\title{
EL AGRUPAMIENTO DE TEXTOS, UNA ESTRATEGIA PARA LA FORMACIÓN LITERARIA EN FRANCÉS COMO LENGUA EXTRANJERA
}

\author{
TeXt Clustering AS A STRATEgy TO DEVELOP EFL LANGUAge LEARNERS' Literary COMPETENCE \\ LE GROUPEMENT DE TEXTES, UNE STRATÉgie POUR LA FORMATION LitTÉraire EN FranÇAis LANGUE \\ ÉTRANGÈRE
}

\begin{abstract}
Andrés Iván Bernal Vargas
Docente de Francés como Lengua

Extranjera y Literatura, Escuela de

Ciencias del Lenguaje, Universidad del Valle, Cali, Colombia. Magíster en Lingüística y Español, y miembro del grupo de investigación en Interculturalidad, literatura y lengua (Interclé), Universidad del Valle, Cali.

Carrera 100 N. $^{\circ} 45-51$, C. P. 760026, B/ Corales del Lili, Cali, Valle, Colombia andres.bernal@correounivalle.edu.co
\end{abstract}

El presente artículo es producto de la investigación "El agrupamiento de textos, una estrategia transversal para la formación literaria en francés lengua extranjera", financiada por la Universidad del Valle, y registrada en Vicerrectoría de Investigaciones con el código $\mathrm{Cl}-4330$, en el marco de una convocatoria interna. Esta investigación se realizó entre agosto de 2014 y junio de 2016.

\section{RESUMEN}

La comprensión de lectura y la escritura de textos son dos procesos académicos complejos para estudiantes de lenguas extranjeras. Ambos procesos pueden ser articulados por medio del agrupamiento de textos (AT) como estrategia didáctica en la enseñanza de lenguas. Este artículo reporta una investigación-acción de tipo mixta que implementó el AT para desarrollar tanto la comprensión de lectura y la escritura, como la competencia literaria, en treinta y tres jóvenes aprendientes de francés de una licenciatura en lenguas extranjeras en una universidad colombiana. El estudio duró dos años. Se hizo inicialmente un análisis documental de los programas de los cuatro primeros niveles de francés del currículo, constatándose que no se explicitaban ni objetivos ni contenidos del componente de literatura. Se administró, además, una encuesta de entrada y salida para identificar los conocimientos (previos y finales, respectivamente) sobre elementos esenciales de la narración. Durante la intervención pedagógica se implementó el AT (dos cuentos y una película) para que los estudiantes reconocieran la singularidad genérica del cuento, siguiendo el esquema narrativo de Larivaille (1974) y el actancial de Hamon (1991), y produjeran, posteriormente, textos literarios de este género. El estudio concluyó que el AT fue efectivo, pues 1) permitió integrar un componente literario a las clases de francés para nivel B1, y 2) la mayoría de estudiantes mejoraron sustancialmente sus procesos de lectura y escritura de cuentos.

Palabras clave: competencia literaria, agrupamiento de textos, esquema narrativo del cuento, lectura y escritura en francés como lengua extranjera, escritura de invención, enseñanza de lenguas extranjeras

\section{Abstract}

Reading and writing are two complex academic processes for foreign language learners. These processes can be integrated through text clustering (TC) as a language teaching strategy. This article reports on an action research with a mixedmethods design that implemented TC to developing reading, writing and literary competence in 33 young French language learners ( 22 years old average) in a B. A. in Foreign Languages programme at a Colombian university. The study was conducted for two years. Initially, a documentary analysis of four French courses syllabi was carried out, confirming that neither objectives nor contents of the literature

Received: 2017-04-30 /Accepted: 2017-10-13

DOI: $10.17533 /$ udea.ikala.v23n03a05 
component were explicitly stated. In addition, an entrance and exit surveys were administered to identify previous and final knowledge on basic narration features. During the pedagogical intervention, TC (two short stories and a film) was implemented so that the students could identify genre (narration) particularities following Larivaille (1974) and Hamon (1991) and produce narrative texts. It is concluded that TC was effective, first, because it allowed to integrate a literary component in the French language course for B1 level learners, and second, because most students improved narrative reading and writing.

Keywords: literary competence, text clustering, narrative scheme, reading and writing in French as a foreign language, foreign language teaching, creative writing

\section{RÉSUMÉ}

La compréhension et la production écrites sont deux processus académiques complexes pour les étudiants en langues étrangères. Ces deux processus peuvent s'intégrer grâce au groupement de textes (GT) comme stratégie didactique dans l'enseignement de langues. Cet article rapporte une recherche-action de type mixte qui met en œuvre le GT afin de développer la lecture, l'écriture et la compétence littéraire chez 33 jeunes apprenants de français d'une licence en Langues Étrangères d'une université colombienne. L'étude a été menée pendant deux ans. On a fait d'abord une analyse documentaire des programmes des quatre premiers niveaux de français et on a pu constater que les objectifs et les contenus du composant littéraire n'étaient pas explicites. De plus on a fait un sondage avant et après l'expérience pédagogique pour repérer les connaissances préalables puis finales sur des éléments essentiels de la narration. Durant l'intervention pédagogique, on a mis en œuvre le GT (deux contes et un film) pour que les étudiants puissent identifier la singularité générique du conte d'après le schéma narratif de Larivaille (1974) et l'actanciel de Hamon (1991) et produisent, ensuite, des textes littéraires du même genre. L'étude a conclu que le GT a été efficace car, d'abord, il a permis l'intégration du composant littéraire dans les cours de français niveau $\mathrm{B} 1$, et enfin, la plupart des apprenants ont amélioré leurs processus de lecture et d'écriture de contes.

Mots clés : compétence littéraire, groupement de textes, schéma narratif du conte, lecture et écriture en français langue étrangère (FLE), écriture d'invention, enseignement de langues étrangères 


\section{Introducción}

El trabajo del autor de la investigación que aquí se presenta, como docente de francés como lengua extranjera (FLE) en dos universidades colombianas (Universidad del Valle y Universidad Santiago de Cali), le permitió evidenciar las serias dificultades que los estudiantes experimentan en el campo de las competencias escritas, tanto en la comprensión (lectura) de documentos escritos en dicho idioma, como en su producción. De ahí su propósito de ocuparse, con especial atención, del desarrollo de la lectura y la escritura en el aprendizaje del francés para la formación de futuros licenciados.

En este artículo se expone, inicialmente, la problemática que existe en torno a la promoción complementaria de los procesos de comprensión y producción escrita en FLE, de forma que se permita, a la vez, el desarrollo de la competencia literaria.

Luego, se enuncia en qué consiste la estrategia del agrupamiento de textos, que permite trabajar la comprensión de cuentos y el reconocimiento de su esquema narrativo (Larivaille, 1974) y actancial (Hamon, 1991), con el fin de plantear a los estudiantes, posteriormente, la creación de un cuento corto. Para ello se siguen, además, las propuestas de los teóricos de la escritura de invención (Genette, 1982; Houdart-Merot, 2004).

Después se enuncia la metodología, que consiste en una investigación de tipo mixto, orientada en la investigación-acción, propuesta a estudiantes de FLE de segundo año que iniciaban su formación en el nivel B1 de la Licenciatura en Lenguas Extranjeras, de la Universidad del Valle.

Al final, se evalúa el impacto de la propuesta investigativa y se sugiere extender la iniciativa a los otros cursos del nivel básico de formación de la licenciatura.

\section{Planteamiento del problema}

Frente a la cuestión de las competencias principales que en cultura escrita en lengua extranjera, como son la lectura y la escritura, requiere adquirir y desarrollar un estudiante, se necesita una propuesta didáctica que las sepa focalizar de manera conjunta y logre darles un tratamiento que permita ver su progreso, a la vez que favorezca la formación literaria. Asociar en una misma estrategia formativa la lectura y la escritura ya se emplea en Francia en las clases de francés (lengua materna) $y$, particularmente, en la enseñanza de la literatura en secundaria, a partir de lo que se denomina el agrupamiento de textos (groupement de textes). La hipótesis, entonces, es que esta perspectiva puede ser de gran beneficio para la enseñanza de dichas competencias en FLE en el contexto colombiano, en el plano de la formación de licenciados de lenguas extranjeras.

En el presente proyecto se abordó, entonces, un agrupamiento de textos que se orientó al reconocimiento del esquema narrativo del cuento como eje de la secuencia didáctica, en búsqueda del desarrollo de las competencias escritas (de comprensión y de producción) y que estaría más acorde con su formación literaria, pues abordar la literatura en los primeros semestres de una licenciatura no debe limitarse a la simple lectura de obras cortas o de fragmentos literarios, como ha sido la experiencia en nuestro programa de Lenguas Extranjeras, dada la falta de formación en literatura de quienes orientamos normalmente los niveles de FLE.

\section{Pregunta de investigación}

En el ámbito de la enseñanza de la literatura en modalidad de FLE para licenciados en Colombia, ¿sería el agrupamiento de textos una estrategia exitosa que asocie la comprensión y la producción de textos, al tiempo que desarrolle la competencia literaria de los estudiantes?

\section{Objetivos}

\section{General}

Implementar el agrupamiento de textos y evaluar si es una estrategia exitosa que asocie la 
comprensión y la producción de textos, al tiempo que desarrolle la competencia literaria de los estudiantes del curso de Habilidades Integradas IV del programa de Licenciatura en Lenguas Extranjeras de la Escuela de Ciencias del Lenguaje de la Universidad del Valle.

\section{Específicos}

1. Describir los objetivos y los contenidos de los programas de las asignaturas de Habilidades Integradas en Francés (HIF) de los semestres I a IV en términos del componente de literatura.

2. Proponer a dosgrupos del curso de Habilidades Integradas IV un agrupamiento de textos que ofrezca la lectura de cuentos para estudiar las particularidades de este género literario y de las técnicas del relato.

3. Orientar y acompañar el proceso de escritura de invención, en la redacción de un cuento corto.

4. Evaluar las capacidades de los estudiantes para identificar el esquema narrativo y el actancial de los cuentos propuestos en el agrupamiento de textos, al igual que sus capacidades para replicar tales esquemas en la redacción de nuevos cuentos.

\section{Marco teórico}

En este apartado se exponen los conceptos claves: competencia literaria, agrupamiento de textos, narratología, semiótica narrativa y escritura de invención, que dan el soporte teórico a la presente investigación.

\section{La competencia literaria}

Según la propuesta del Ministerio de Educación Nacional (MEN) de Colombia, en su publicación de los Lineamientos curriculares lengua castellana, la competencia literaria se concibe como

[...] la capacidad de poner en juego, en los procesos de lectura y escritura, un saber literario surgido de la experiencia de lectura y análisis de las obras mismas, y del conocimiento directo de un número significativo de éstas (Colombia, MEN, 1998).

Desde el campo de los profesores de literatura, añade el MEN (1998), son ellos los encargados de definir los criterios para la selección de los textos, a partir de su propia competencia literaria.

La competencia literaria del docente atravesaría dos dimensiones: 1. el reconocimiento del canon literario (las obras representativas en la diacronía de la literatura), y 2. la apropiación de enfoques teórico-analíticos (semiótica, filosofía, estética, historiografía, sociología, retórica, etcétera). Esta segunda dimensión es determinante para trasvasar las impresiones primarias de las obras hacia la búsqueda de las lógicas de sentido que las constituyen: ir siempre de unas lecturas primarias hacia lecturas más profundas en donde se pone en juego la red de saberes que la escuela, entre otros espacios, ha proporcionado. Las dos dimensiones alcanzan su dinámica en el foro, en el debate y en la producción escrita (p. 57).

Ahora bien: el proceso de lectura debe concebirse y producirse de manera concomitante al proceso de escritura, lo que requiere, indudablemente, de la participación decidida del aprendiente para que podamos considerarlo, entonces, como un lectorescritor. En palabras de Séoud (1997):

El acto de lectura [...] es percibido desde ahora, como un acto de creación, o mejor, de creatividad. Es por la lectura que el texto, un texto, se crea; cada lectura aparece como una nueva escritura del texto. Leer y escribir se confunden, el tema de la lectura-escritura se convierte rápidamente en un tema dominante (p. 162). ${ }^{1}$

En la misma dirección, Mekhnache (2010) afirma que la lectura no puede ser vista como un proceso lineal de descubrimiento de texto, sino, más bien, como un proceso integrativo, que puede lograrse solamente con el consentimiento del aprendiente,

1 La traducción es del autor. El original dice: "L'acte de lecture [...] est désormais perçu comme un acte de création, ou mieux, de créativité. C'est par la lecture que le texte, un texte, se crée; chaque lecture apparaît comme une nouvelle écriture du texte. Lire et écrire se confondent, le thème de la lecture-écriture devient vite un thème dominant." 
es decir, puede haber acto de lectura a través del placer de leer para poder escribir.

En nuestro ejercicio docente es fácil identificar en algunos estudiantes su deseo de escribir. En ocasiones, son ellos quienes se acercan al profesor con un párrafo, un pequeño texto, o un poema, de su propia autoría, con el fin de solicitar una lectura y recibir a cambio sugerencias y comentarios sobre dicha producción. Este tipo de experiencias las anota, igualmente, Séoud (1997), quien manifiesta, además, que un aprendiente de FLE al que no le agrade aprender a analizar un texto, puede, sin embargo, escribir y crear sus propios textos, en respuesta a sus propios gustos, sin olvidar sus obligaciones escolares.

Asimismo, Séoud (1997, p. 163) cita a Ricardou quien, en Le Colloque de Strasbourg, cuestiona la brecha pedagógica entre el proceso de comprensión y el proceso de producción, entre discurso sobre la literatura y práctica de la literatura. Posteriormente, Ricardou (en Séoud, 1997, p. 164) añade que esta práctica debe ser asidua y que la escritura debe ser tan normal como la práctica de la lectura.

En esta misma perspectiva, Leclair, en "Autour du poème: Former de vrais lecteurs (About the Poem: Creating True Readers)" (1992), subraya la exigencia de unir la escritura a la lectura, pues la práctica de la primera conduce al aprendizaje de la segunda. Así, la buena lectura es la que implica la escritura.

Desde la perspectiva del agrupamiento de textos, Moreno (2001) expresa: "la competencia literaria, [...] debe ser vista en la triple dimensión de lectura, apropiación y argumentación” (p. 118). En otras palabras, el AT permite la formación literaria, toda vez que integra las competencias escritas de comprensión y producción.

Por consiguiente, la competencia literaria debe ser entendida como la necesidad no solo de leer, sino también de escribir, es decir, comprender, pero también crear, producir.

\section{El agrupamiento de textos}

El AT es concebido, primordialmente, como una actividad de lectura. Boissinot y Mougenot mencionan que

[...] las Instrucciones oficiales [en Francia] tienen razón al recordar con insistencia que si bien el agrupamiento de textos puede solicitar una temática [para su conformación], éste es ante todo el lugar donde la confrontación de los textos hace surgir una problemática literaria (1992, p. 4). ${ }^{2}$

Estos autores señalan, más adelante, que practicar el AT es esencialmente una forma de lectura metódica totalmente formadora (Boissinot y Mougenot, 1992, p. 5). En otras palabras, el AT es una estrategia didáctica que permite asociar diferentes textos literarios (de distinto género o del mismo), que tienen un asidero coyuntural en torno a un fenómeno sociológico o sicológico, a un problema teatral, a un tema poético, a una estructura narrativa, etc.

El AT para la lectura, según Jordy (1992),

[...] es un recurso didáctico, una técnica para responder a las exigencias del aprendizaje de la lectura autónoma de textos, sin importar su naturaleza, su base o su tipo. [...] Los agrupamientos, lejos de ser conjuntos aventureros de elementos misteriosamente asociados, deben componerse de series cuya combinación y orden tejan una sólida coherencia que se debe menos a los textos en sí mismos que a la voluntad de quien dispuso tal coyuntura, consciente de los objetivos de aprendizaje que tiene en mente (p. 10). ${ }^{3}$

2 La traducción es del autor. En francés dice: "les Instructions officielles ont raison de rappeler avec insistance que le groupement de textes, s'il peut solliciter une thématique, est avant tout le lieu où la confrontation des textes fait émerger une problématique littéraire".

3 La traducción es del autor. El original dice: "est un instrument didactique, une technique pour répondre aux exigences de l'apprentissage de la lecture autonome des textes, quels que soient leur nature, leur support ou leur type. [...] Les groupements, loin d'être des rassemblements aventureux d'éléments mystérieusement associés, doivent composer des séries dont la combinaison, l'ordonnancement tissent une cohérence solide qui doit moins aux textes eux-mêmes qu’à la volonté de 
A su vez, el AT consta de cuatro tipologías:

a. El AT utilizado para poner en marcha y garantizar el manejo de instrumentos de análisis textual.

b. El AT empleado para consolidar el reconocimiento y la distinción de las "clases" de textos: formas, géneros, tipos, registros.

c. El AT para construir saberes de orden cultural sobre un autor, un periodo, un movimiento estético.

d. El AT en sus relaciones con la obra integral para extender y preparar el estudio, para profundizar sobre un aspecto relevante en un trabajo intertextual.

De hecho en todo proyecto coherente, no hay AT que no tenga esta cuádruple dimensión. [...] Claro está que es el enseñante quien, a partir de los objetivos propuestos para su curso, debe precisar la prioridad para cada AT (p. 11).

A manera de ejemplo, se ilustra a continuación una propuesta que combina las tipologías b y c. Esta es una de las sugerencias hechas en Francia por el Ministère de l'éducation nationale, en "Enseignements élémentaire et secondaire", del Bulletin officiel $n^{\circ} 46$ du 14 décembre 2006, programa de enseñanza general en francés en grado $2{ }^{\circ}$ general y tecnológico, y en grado $1 .^{\circ}$ de series generales $\left(100^{\circ} \text { y } 11 .^{\circ} \text { para el sistema colombiano }\right)^{4}$ y programa de enseñanza de literatura en grado $10^{\circ}$, con énfasis literario. ${ }^{5}$ En este boletín se recomienda abordar los contenidos de géneros y las formas de la argumentación en los siglos XVII y XVIII, y se propone el siguiente corpus: "Un texto largo o un conjunto de textos que tengan una fuerte unidad: capítulo de novela, libro de fábulas, colección de sátiras, cuento filosófico, ensayo o parte de ensayo, según la elección del profesor" ${ }^{6}$

celui que a arrangé cette conjoncture, conscient des objectifs d'apprentissage qu'il vise".

4 Aunque, en Francia, la secundaria va hasta el grado 12.

5 La traducción es del autor. El original dice: "Programme de l'enseignement commun de français en classe de seconde générale et technologique et en classe de première des séries générales et programme de l'enseignement de littérature en classe de première littéraire”.

6 La traducción es del autor, del original: "Un texte long ou un ensemble de textes ayant une forte unité : chapitre de roman, livre de fables, recueil de satires, conte philosophique, essai ou partie d'essai, au choix du professeur."
Así, pues, el AT es una estrategia para la formación literaria en FLE, en la medida en que los corpus elaborados respondan a las búsquedas propuestas en los objetivos en cada uno de los niveles de FLE.

Ahora, el AT, una estrategia para la formación literaria, se constituye de varias tareas para su ejecución: la formulación de módulos, la construcción del corpus y la construcción del modelo operativo de la estrategia didáctica.

- Formulación de módulos y construcción del corpus. Para estas tareas, Lager (2003) señala:

[...] principio importante es la organización por "groupement de textes" o módulos que agrupan textos en torno a una temática. Se debe evitar el estudio de textos aislados, sacados de su contexto. Lo aconsejable es construir secuencias que agrupen textos de varios autores; de varios géneros, que ofrezcan una pluralidad de puntos de vista, que permitan estudiar varias técnicas literarias (p. 196).

Así pues, en el corpus creado para la presente investigación, se propuso trabajar en torno a la temática del género narrativo, precisamente en el reconocimiento de la singularidad genérica del cuento. $^{7}$

Valga precisar que un corpus puede contar con tres, cuatro o más textos, bien sea completos o fragmentos de los mismos, y su constitución depende de las intenciones pedagógicas del docente.

- Construcción del modelo operativo de la estrategia didáctica. Siguiendo la iniciativa de concebir una estrategia integral para la enseñanza y el aprendizaje de la competencia literaria, que en la fase de lectura implemente el agrupamiento de textos (de cuentos para el caso de nuestra investigación) y en la fase de escritura implemente la redacción de cuentos,

7 Esto se amplía en los párrafos siguientes, y se ilustra en la sección "Instrumentos" del apartado "Metodología". 
se hace necesaria una descripción-explicación del modus operandi en el contexto de la práctica formativa y evaluativa.

Se tiene, entonces, que, para cada texto del agrupamiento, debe diseñarse un documento que permita, además de verificar la comprensión del cuento, identificar las etapas que constituyen su esquema narrativo y los personajes y roles que hacen parte del esquema actancial. Elemento central de este modelo es, pues, la creación de la situación didáctica (véase ejemplo en el anexo 1).

Entonces, ¿cómo integrar las competencias de lectura y escritura en una misma dirección?

En resumen, el desafío que proponemos para el desarrollo de la competencia literaria es servirse del agrupamiento de textos, en una primera fase, para el reconocimiento de las características esenciales del género (del cuento para nuestro caso), y posteriormente, en la segunda fase de producción escrita, la transposición de las particularidades identificadas en la etapa inicial para la creación de textos literarios del mismo género.

Ahora, la experimentación literaria de integración de la lectura y la escritura en torno al género narrativo tendría su fundamentación teórica, por un lado, en la narratología y la semiótica narrativa, y por otro, en la escritura de invención.

\section{Narratología y semiótica narrativa}

En la visión de Jouve (2006), mientras la narratología se encarga de las estructuras del relato, la semiótica narrativa se ocupa, de manera paralela, de las estructuras de la historia. En tanto que la primera analiza el material utilizado para contar, la segunda estudia lo que se cuenta. Para la semiótica, la historia, de manera mínima, puede ser definida como una secuencia de acciones a cargo de actores. Para aclarar estos aspectos centrales a la semiótica, abordamos entonces la intriga y los personajes.

\section{La intriga}

Jouve (2006) expone, en La poétique du roman, que la semiótica narrativa parte de un principio esencial: todas las historias se parecen, sin importar el lugar y la época en la que nacieron. El ruso Vladimir Propp (1928, citado en Jouve, 2006, p. 46), asume que, a pesar de sus diferencias, todos los relatos maravillosos se asemejan, pues tienen una base común de treinta y una funciones que proporcionan una estructura lógica a la intriga.

Con el tiempo, los estudiosos de Propp han intentado simplificar su propuesta, y el modelo estructural que se impone actualmente entre las investigaciones de diferentes teóricos es el esquema quinario de Paul Larivaille, de 1974. Para él, toda historia se reduciría a una secuencia lógica de cinco etapas. La intriga respondería, entonces, al siguiente modelo:

1. Antes - Estado inicial - Equilibrio.

2. Provocación - Detonante - Disparador.

3. Acción.

4. Sanción-Consecuencia.

5. Después - Estado final - Equilibrio.

Según Jouve (2006, p. 47), este modelo, que explica la secuencia narrativa elemental de los cuentos, sirvió de manera muy eficaz para establecer la lógica profunda que subyace a la intriga de todo relato, y agrega que el relato se define como el paso de un estado a otro.

\section{Los personajes}

Jouve (2006) señala, igualmente, que:
El personaje es el segundo objeto de estudio privilegiado por la semiótica. Así como se considera que toda historia tiene un modelo lógico relativamente simple, la crítica de tendencia greimasiana piensa que en la gran variedad de relatos se puede reconocer el mismo sistema de personajes. En realidad, todo relato basado en un conflicto cuenta con al menos dos personajes presentes en toda novela: el sujeto y su adversario (p. 51). ${ }^{8}$

8 La traducción es del autor. El texto original dice: "Le personnage est le deuxième objet d'étude privilégié par la sémiotique. De même qu'elle considère qu’on peut ra- 
Así pues, lo que hace el personaje (su recorrido) y esencialmente lo que es el personaje (su retrato) se analiza en el modelo semiótico propuesto por Philippe Hamon (1991).

Es necesario empezar por precisar que la noción de "personaje" no existe para la semiótica narrativa. Dicha noción es remplazada por tres conceptos que intervienen en niveles diferentes de descripción del relato: el actor, el actuante y el rol temático (Jouve, 2006, p. 51).

- El actor: es el concepto más cercano a la noción tradicional de "personaje". Para que un relato funcione necesita algunas acciones y es el actor el encargado de ejecutarlas. Es la encarnación antropomorfa de los roles necesarios para el desarrollo del relato.

- El actuante: es una noción construida a partir del análisis y no un dato del texto. El actuante se define como un rol necesario para la existencia del relato, es decir, el rol que representan los actores. Según Greimas (citado en Jouve, 2006, p. 52), los actuantes o papeles actanciales son seis: sujeto, objeto, oponente, ayudante, destinador (solicitante) y destinatario. Este esquema actancial lo representan Adam y Lorda (1999) a través de tres relaciones, así:

1. Relación de deseo (querer) o de búsqueda: Sujeto $\rightarrow$ Objeto de valor

2. Relación de comunicación (saber):

Destinador $\rightarrow$ (Sujeto $\rightarrow$ Objeto)

$\rightarrow$ Destinatario

3. Relación de lucha (poder):

Adyuvante $\rightarrow$ (Sujeto $\rightarrow$ Objeto)

$\leftarrow$ Oponente

Este esquema indica que el sujeto-héroe (o protagonista) se lanza en busca de un objeto va-

mener toute histoire à un modèle logique relativement simple, la critique de tendance greimassienne pense qu'on peut retrouver dans l'infinie pluralité des récits le même système de personnages. De fait, tout récit étant fondé sur un conflit, il est au moins deux personnages présents dans tout roman : le sujet et son adversaire". lorado por el destinador (relación de deseo: querer), cuya existencia y valor le ha revelado el destinador, convertido en informador (relación de comunicación: saber). En su búsqueda, el sujeto-protagonista puede ser ayudado por colaboradores/adyuvantes, o combatido por adversarios/oponentes (relación de lucha:poder) (p.25).

Ahora, Jouve (2006, p. 53) señala que así como un actor, único, puede desempeñar varios roles actanciales, diferentes actores pueden agruparse en un solo actuante. Además, un actor puede bien sea cambiar de rol actancial, modificar su programa narrativo o seguir varios.

- El rol temático: el actor es visto desde el plano figurativo, o sea, como portador de un "sentido". El rol temático contempla las características psicológicas (la amable, el hombre infiel, el deshonesto, etc.) o sociales (la profesora, el vendedor, el deportista, etc.) que permiten reconocer al personaje en el plano del contenido. Si el rol actancial garantiza el funcionamiento del relato, el rol temático le permite vehicular el sentido y los valores, y así definir su alcance ideológico.

Tenemos, entonces, que el esquema narrativo quinario de Larivaille (1974) y el modelo semiótico de Hamon (1991) (actor, actuante y rol temático) se constituyen en el piso teórico de nuestra propuesta de producción escrita, en cuanto a la creación de un cuento se refiere.

\section{Escritura de invención}

Según Ouellet y Boutin (2006), hay investigaciones que demuestran que la escritura de invención realizada a partir de "modelos" literarios sobre autores reconocidos y obras representativas, permiten al estudiante alcanzar aprendizajes significativos, y que la escritura literaria puede, entonces, ser vista como un ejercicio de consolidación de conceptos literarios estudiados.

En esta perspectiva, para Genette (1982), la imitación o pastiche de una obra en particular, un autor 
específico, una escuela, una época o un género, son operaciones idénticas en su estructura. Para Houdart-Merot (2004), por el contrario, un pastiche de escritor o de escuela presupone la imitación no solo de ese autor o esa escuela, sino también su visión del mundo y sus valores. Agrega que las imitaciones de género suscitan otras formas de reescritura que no están centradas en las constantes de temas o estilos, sino en el conocimiento de las reglas y de las convenciones asociadas a tal o cual género. Esta autora subraya que, de manera más general, se puede considerar que todo texto lleva consigo relaciones con las formas genéricas que le precedieron y con las reglas implícitas constituidas a través de la lectura de diferentes textos. Como ella misma lo dice: "Es esta relación con códigos o con modelos que Gérard Genette llama 'architextualidad'. [...] Además, el conocimiento de esos códigos y de esos modelos es indispensable tanto en una perspectiva de lectura como de escritura” (p. 43).

Ahora, si hablamos de reescritura, es necesario mencionar también la crítica genética. Esta ciencia, reciente por demás, propone nuevas perspectivas tanto para la interpretación de textos literarios como para el conocimiento de las prácticas de escritura y de reescritura. La crítica genética se ocupa, pues, del proceso de escritura, de la obra como productividad y no como producto. Para el teórico Almuth Grésillon (citado en Houdart-Merot, 2004, p. 45), toda escritura, cualquiera que sea y de donde quiera que venga, conoce cuatro operaciones de reescritura: agregar, suprimir, remplazar y permutar. Asimismo, la profesora Houdart-Merot las reconoce como cuatro modos de reescritura: inserción de textos, transformación de un texto singular, imitación de la manera de escribir o de discurso y, por último, imitación de géneros o de registros.

Señalamos la estrecha relación de esta última operación, la imitación de géneros, y más particularmente, del pastiche de género como parte de dicha operación, con la dirección dada a la producción escrita en la presente investigación.

Insistimos, pues, en lapropuestadeque elestudiante logre crear un cuento corto, producto del reco- nocimiento previo de sus particularidades como género, por medio del acercamiento a otros cuentos presentados en el agrupamiento de textos. El pastiche refuerza tal idea, pues, según Houdart-Merot (2004, p. 172), la imitación procura reproducir un género o un registro a partir de un conjunto de textos y no de un texto en particular.

Finalmente, Houdart-Merot destaca que los mismos procesos de reescritura están en la obra y estos suponen que quien escribe se haga lector, lector crítico y "corrector" de los escritos de otro y de sus propios escritos (2004, p. 46).

\section{Metodología}

\section{Contexto}

Esta investigación se realizó entre agosto de 2014 y junio de 2016 con 33 jóvenes (edad promedio de 22 años), matriculados en cuarto semestre de la Licenciatura en Lenguas Extranjeras de la Universidad del Valle, en el curso de FLE que iniciaban su formación en el nivel B1.

\section{Desde la investigación-acción}

El presente estudio es de tipo mixto, ya que alterna aspectos de corte cualitativo en su mayoría y aspectos cuantitativos (explicados en el apartado "Instrumentos"), y se inscribe en la investigación-acción.

Desde la perspectiva de Elliott (1993), la investigación-acción es

[...] el estudio de una situación social para tratar de mejorar la calidad de la acción en la misma. Su objetivo consiste en proporcionar elementos que sirvan para facilitar el juicio práctico en situaciones concretas y la validez de las teorías e hipótesis que genera no depende tanto de pruebas "científicas" de verdad, sino de su utilidad para ayudar a las personas a actuar de modo más inteligente y acertado (p. 88).

En ese sentido, cuando la investigación acción se enfoca a la práctica educativa o a la sociológica, busca proporcionar información que guíe la toma 
de decisiones y los procesos de cambio para mejorar la misma.

Por su parte, Sandín (2003, p. 43) señala que otro de los aspectos fundamentales que caracteriza a la investigación-acción es su interés no solo por el proceso, sino también por el producto, es decir, que a través del proceso se pretende mejorar la práctica y se considera, igualmente, que el camino a recorrer para conseguirlo es tan importante o más que el resultado final. "El cambio es un proceso, no un producto", afirman Kemmis y McTaggart (1988, citados en Sandín, 2003, p. 43).

\section{Instrumentos}

Se llevó a cabo, inicialmente, un análisis documental, con el fin de identificar y describir los objetivos y contenidos de los programas de las asignaturas de HIF de los semestres I a IV en términos del componente de literatura.

Posteriormente, se aplicó una encuesta de entrada, adaptada de Le Conte Merveilleux (s. a.) (véase anexo 2), encaminada a detectar los saberes literarios puestos en juego en el estudio y, en particular, a examinar los conocimientos previos de los estudiantes en cuanto a las características esenciales del cuento.

Luego se dio paso a la intervención pedagógica (agosto a diciembre de 2014), que se ilustra a continuación con la estrategia del agrupamiento de textos. Para la primera fase:

- Formulación de módulos. Dada la inexistencia de contenidos y de objetivos para la formación literaria en los cuatro cursos del ciclo básico de formación del programa de Lenguas Extranjeras y como recurso sustancial de la presente investigación, se propusieron módulos para la enseñanza y el aprendizaje de la competencia literaria en FLE (véase tabla 1 en el apartado "Resultados"). Se formuló, entonces, para el curso de Habilidades Integradas en Francés IV una temática que posibilitara enfocarse en el género narrativo, específicamente en las particularidades del cuento.

Tabla 1 Corpus sugeridos para la enseñanza y el aprendizaje de la competencia literaria en FLE en los cuatro cursos del ciclo básico de formación del programa de Lenguas Extranjeras

\begin{tabular}{lll}
\hline Curso y semestre & Módulo & Agrupamiento de textos \\
\hline Habilidades Integradas en Francés I & Introducción al teatro: escenas de comedia & $\begin{array}{l}\text { Faites l'amour... pas des gosses (S. } \\
\text { Depooter et S. Judaszko) } \\
\text { Le fusible (S. Meyniac) } \\
\text { Le dîner des cons (F. Veber) }\end{array}$ \\
Habilidades Integradas en Francés II & La novela francófona para estudiantes de FLE. & Trois versions de la vie (Y. Reza) \\
& Premio de cine: Festival de Cannes & Le festival n'aura pas lieu (G. Jacob) \\
Habilidades Integradas en Francés III & Les faits divers y la historia policíaca (Polar). La & Le père de nos pères (S. Weber). \\
intriga. Fragmentos de obras & L'Affaire du tarot(P. Aspe) \\
Habilidades Integradas en Francés IV & El cuento y sus esquemas: narrativo y actancial & Article I22-l (D. Messager) \\
& & Hansel et Gretel(Hermanos Grimm) \\
& & La mère Noël(M. Tournier) \\
& & Kirikou et la sorcière (Película animada de \\
\end{tabular}

Fuente: Elaboración propia. 
- Construcción del corpus. Nuestro proyecto se dirigió a estudiantes de cuarto semestre que habían iniciado un nivel B1 en su formación en FLE, es decir, que los jóvenes ya contaban con los recursos lingüísticos para afrontar la lectura de textos de género narrativo como una tarea acorde con sus posibilidades académicas. En vista de lo anterior, propusimos integrar, en un mismo corpus, los siguientes textos, en los que se pueden identificar fácilmente el esquema narrativo y el actancial: dos cuentos, "Hansel et Gretel", de los hermanos Grimm (1986), y "La mère Noël” (Tournier, 2008); el AT se complementó con la película animada Kirikou et la sorcière (Ocelot, 1998).

- Construcción del modelo operativo de la estrategia didáctica. Para cada uno de los tres textos del corpus se diseñó un taller que permitió, gracias a la puesta en común, verificar la comprensión de las historias y el reconocimiento de los esquemas (narrativo y actancial) característicos del cuento (véase ejemplo en el anexo 1, que ilustra el trabajo hecho con el cuento "Hansel y Gretel", que, para efectos del taller, se entregó fraccionado).

Adicionalmente, se trabajaron dos recursos como soporte teórico a la situación didáctica: una presentación de diapositivas (elaborada a partir de contenidos de Alloprof, 1996) y un crucigrama (tomado del blog de Pineau, 2012). Estos elementos ilustraron el esquema quinario de Larivaille (1974) y el esquema actancial de Hamon (1991).

Posterior a la lectura de cuentos y durante la segunda fase de la intervención pedagógica, se propuso a los estudiantes redactar un cuento (de manera individual o en pareja). La tarea fue, pues, transponer las características esenciales de este género narrativo, trabajadas en el AT durante la fase inicial, en la invención de su propio cuento.

La evaluación del proceso de escritura vivió igualmente varios momentos, ya que cada cuento fue escrito por los estudiantes al menos tres veces, es decir, dos borradores de la historia antes de generar el texto final. En primera instancia se recurrió a la coevaluación; así, cada estudiante-escritor o cada pareja, leyó dos cuentos para hacer sugerencias de orden lingüístico o de redacción, y corregir las producciones. Luego, un segundo borrador se entregó al docente, con el fin de hacer recomendaciones para seguir mejorando el documento y proceder, al final, a la reescritura de la versión final del cuento.

La evaluación de dicha versión tuvo en cuenta, además de la entrega puntual de los borradores, otros criterios como: el manejo de los aspectos literarios del módulo formulado, es decir, la identificación de las etapas del esquema narrativo y los elementos mínimos del esquema actancial; el empleo de conectores lógicos que brinden coherencia y cohesión a la historia; la creatividad-originalidad del cuento y, finalmente, componentes de orden lingüístico (léxico, morfosintaxis).

Para terminar, se aplicó una encuesta de salida, que fue la misma encuesta de entrada (véase el anexo 2), para verificar el progreso de los estudiantes en el reconocimiento de los elementos que caracterizan el cuento y su esquema narrativo.

\section{Resultados}

Exponemos a continuación los resultados, agrupados en tres momentos: diagnóstico, intervención pedagógica y evaluación.

\section{Diagnóstico}

\section{Análisis documental}

Para empezar, la revisión de los diferentes programas de los cursos de HIF de los semestres I a IV en el programa de Lenguas Extranjeras no arrojó como resultado de manera explícita ni objetivos ni contenidos que propusieran el desarrollo del componente de literatura. En algunos programas se encontró, ocasionalmente, la propuesta de lectura de un mismo libro para el conjunto de la 
clase, sin precisar el procedimiento a seguir. Luego de hablar con dos o tres colegas que orientan los cursos de estos niveles, ellos manifestaron que tal propuesta de lectura se hace según el nivel de los grupos, y sugieren obras de la literatura francesa o, en parte, textos de editoriales que reescriben los libros para facilitar la comprensión de público FLE. La explotación que se hace de dichos documentos se limita, en general, a leer parte de la obra en clase y otra parte como lectura autónoma y, posteriormente, o se socializa con el grupo la trama de la historia o se evalúa de manera sistemática con cuestionarios para verificar su comprensión.

Dicha ausencia explícita de objetivos y de contenidos para abordar el componente literario en los primeros semestres de formación en FLE en el programa de Lenguas Extranjeras nos animó a sugerir algunas ideas para integrar la competencia literaria al aprendizaje del francés, bajo la implementación de la estrategia del AT (véase tabla 1).

Como puede verse en la columna central de la tabla 1 , los corpus propuestos podrían trabajarse como aproximación literaria a géneros como el dramatúrgico y el narrativo (novela y cuento). Además, se pueden plantear objetivos comunicativos que, al igual que los agrupamientos de textos, obedecen a los criterios de cada docente; por ejemplo:

- Complementar el desarrollo de la comprensión y producción oral.

- Abordar contenidos socioculturales.

- Desarrollar la lectura y la escritura de manera conjunta.

- Profundizar en algún aspecto lingüístico.

- Entre otras posibilidades.

\section{Encuesta de entrada}

Esta encuesta permitió identificar que los estudiantes ya poseían un conocimiento previo sobre ciertos aspectos de la narración de un cuento, que se explican a continuación. Así pues, frente a la pregunta inicial sobre el número de etapas del esquema narrativo, de los 33 encuestados, el 78,8\% señaló que eran tres, el 6,1 \% creía que eran 4 etapas, y un $15,2 \%$ reconoció que son 5 etapas.

Asimismo, se pudo constatar que el 93,9\% de los participantes identificó qué es el esquema narrativo; el $87,9 \%$ reconoció qué es la etapa del desarrollo del cuento; el 78,8\% señaló que en un cuento hay al menos dos personajes, y el $72,7 \%$ registró que un cuento muestra que para llegar al objetivo antes es necesario afrontar diferentes tipos de pruebas.

En el apartado de encuesta de salida, que se presenta más adelante, se contrastan los resultados con la encuesta inicial.

Por otro lado, al cuantificar la encuesta, se contabilizaron once preguntas, de la 2 a la 12, y se observó que el 24,2\% del grupo consiguió notas entre 4,1 y 5 , siendo 5 la nota más alta. Por el contrario, el 75,8\% de los estudiantes obtuvo notas poco alentadoras, que oscilaron entre 1,4 y 3,6 . La nota promedio fue de 3,2 entre los 33 estudiantes.

\section{Intervención pedagógica}

La experiencia pedagógica tuvo dos etapas. En la etapa inicial se implementó la estrategia del AT que permitió a los estudiantes leer dos cuentos: "Hansel et Gretel”, de los hermanos Grimm, y "La mère Noël", de Tournier, y ver también la película Kirikou et la sorcière. Ellos lograron, además de comprender las historias, reconocer su esquema narrativo y actancial, gracias a las secuencias didácticas elaboradas para tal fin. A manera de ilustración, se puede consultar el anexo 1 y ver el taller de explotación del cuento "Hansel y Gretel". Asimismo, la presentación del diaporama sobre los esquemas y el trabajo de un crucigrama permitieron consolidar la base teórica del estudio. Luego, en la etapa final, los participantes de la investigación crearon sus propios cuentos, reutilizando los elementos identificados en la fase anterior.

La redacción del cuento fue propuesta y abordada como un proceso que involucró decididamente a la gran mayoría de estudiantes. Los 33 participantes 
optaron por trabajar de manera individual o en parejas, y se recopilaron 23 cuentos.

En el apartado de evaluación que viene a continuación se explica y analiza la producción escrita de los estudiantes.

\section{Evaluación}

1. El cuento. El proceso de producción escrita se caracterizó por la orientación y el acompañamiento permanente entre estudiantes y profesor. Esta etapa permitió a los estudiantes hacer varias modificaciones a sus creaciones, pues los jóvenes elaboraron al menos dos borradores.

Resaltamos que los lectores iniciales del primer borrador fueron sus compañeros, pues se brindó el espacio en clase para que intercambiaran sus producciones. En ese sentido, subrayamos que la coevaluación hizo parte del proceso de creación.

Posteriormente, un segundo borrador se entregó al docente, quien, a su vez, hizo otras recomendaciones escritas y formuló, verbalmente, algunas preguntas al autor o autores de cada cuento, con el fin de aclarar inquietudes sobre la historia. Para terminar, los estudiantes hicieron entrega de la versión final de su texto.

Hay que señalar que la evaluación de los cuentos dejó ver gran creatividad en las historias, manejo normalmente acertado de aspectos lingüísticos, y la implementación y la consolidación de varios elementos literarios que se identificaron en la fase previa de lectura del AT propuesto. En otras palabras, en la gran mayoría de los cuentos $(95,6 \%)$ se evidenció la apropiación de las etapas del esquema narrativo.

Igualmente, un buen número de las creaciones recibidas sobresalieron por su vistosa presentación e incluso por su colorido, ya que estas fueron acompañadas de ilustraciones hechas por los mismos autores, aunque otros se limitaron, simplemente, a tomar imágenes de internet y citar el sitio web de donde fueron extraídas (figuras 1 y 2).

Il aperçut un pot à fleur qui se trouvait pas loin d'où il était et il vola vers la plante sèche, quand il $y$ arriva il picota une fleur fanée et magiquement la fleur tourna vivant avec des couleurs inimaginables.

Figura 1 Cuento “Le colibri à Émeraude”, por Pedro y Claudia

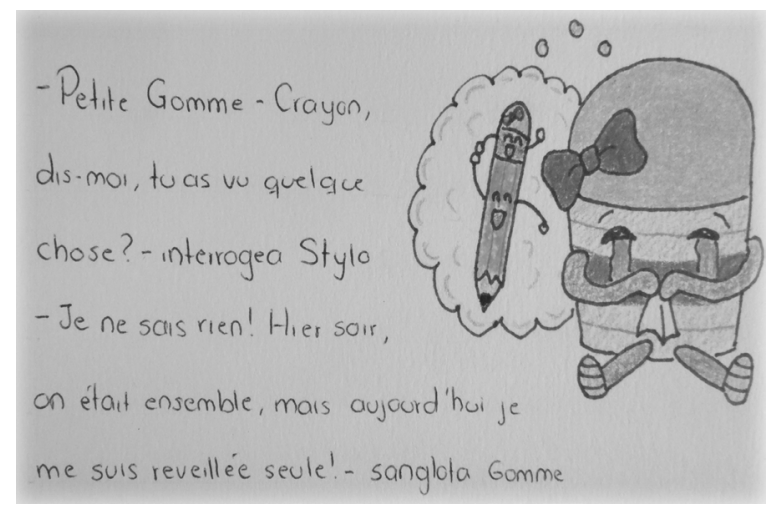

Figura 2 Cuento “Crime d'auteur”, por Darío y Sandra

2. Encuesta de salida. Luego de la intervención pedagógica se aplicó la encuesta de salida, para verificar el aporte de la misma en cuanto al reconocimiento de los elementos esenciales del cuento. Se presentan a continuación los resultados, contrastando las encuestas de entrada y salida.

Para empezar, la respuesta dada a la primera pregunta arrojó un progreso notable. Se indagó por el número de etapas del esquema narrativo del cuento, y se pasó de un 15,2\% de aciertos en la encuesta inicial, a un $81,8 \%$, es decir, de 33 encuestados, 27 respondieron que el esquema 
narrativo se constituye de cinco etapas, aunque, sorprendentemente, un 6,1\% expresó que eran tres etapas, otro $6,1 \%$ dijo que eran cuatro y un $6,1 \%$ señaló, incluso, que eran seis etapas, lo que demuestra que por parte de seis estudiantes $(18,3 \%)$ no hubo una dedicación seria frente al trabajo realizado durante la intervención.

Las preguntas enumeradas de la 2 a la 12 se propusieron bajo la modalidad de selección múltiple, con única respuesta. Sus resultados se agruparon en dos partes: la primera registra modificaciones importantes, porcentualmente hablando, entre los resultados de las dos encuestas, a excepción de la pregunta 2. Y la segunda parte reúne las preguntas 4, 5, 8 y 11 , que no sufrieron grandes cambios.

Así, en el primer grupo tenemos las preguntas 2, 3, 6, 7, 9, 10 y 12. Ante la pregunta 2, que propone definiciones del esquema narrativo, el $94 \%$ de los estudiantes seleccionó, acertadamente, en la encuesta de entrada, que este es una serie de etapas que construyen el cuento y se aumentó ligeramente a un $97 \%$ en la encuesta de salida.

En la pregunta 3, sobre las características de la situación inicial, se obtuvo, en un comienzo, un $48,5 \%$ de aciertos y se pasó a $84,8 \%$ en la encuesta de salida, lo que corresponde a 28 estudiantes de 33 .

Cuando se indagó por el elemento que introduce la situación final, en la pregunta 6 , se pasó de $69,7 \%$ de aciertos a 84,8\%.

Asimismo, con respecto a la pregunta 7 sobre los tiempos más empleados en los cuentos, los estudiantes señalaron que tales tiempos son el pasado simple y el imperfecto, y se pasó de $60,6 \%$ de aciertos a $90,9 \%$.

Ante la pregunta 9, sobre cuál es la búsqueda del héroe, se pasó de 69,7 a 87,9\%.

En el cuestionamiento número 10 se sondeó sobre la ayuda del héroe y se observó una ligera diferencia entre las dos encuestas, donde se pasó de 66,6 a $78,8 \%$.

Finalmente, en este grupo de interrogantes, se mejoró en la pregunta 12 , que indagó por lo que muestra un cuento, y se consiguió un $72,7 \%$ en la encuesta de entrada contra un $81,8 \%$ en la de salida.

Así pues, en síntesis, el incremento en este conjunto de preguntas deja ver que los estudiantes lograron, por un lado, apropiarse de varios elementos que constituyen tanto el esquema narrativo y actancial del cuento, y por otro, reconocer los tiempos más empleados en la redacción de cuentos.

Ahora bien, en lo que respecta el segundo conjunto de preguntas $(4,5,8$ y 11$)$, se agrupan aquellas que no registraron resultados muy diferentes entre la encuesta de entrada y la de salida. Esta baja fluctuación en los resultados se explica, en parte, por un mal diseño de las preguntas y sus respectivas opciones de respuesta, lo que facilitó identificar la alternativa correcta; tal fue el caso de las preguntas 5 y 8 . Ahora, en cuanto a las preguntas 4 y 11 , se aprecia que no hubo apropiación de dos elementos del esquema narrativo y actancial, respectivamente, como se precisa a continuación.

En cuanto a la pregunta 4, que indagó sobre el elemento que perturba la situación inicial, se observó un ligero, pero sorprendente descenso, en el porcentaje de aciertos, y se pasó de 42,4 a $39,4 \%$.

Ahora, la pregunta 5, que interrogó por la definición del nudo o desarrollo de la historia, registró un $87,9 \%$ de aciertos tanto en la encuesta de entrada como en la de salida.

Por su parte, la pregunta 8 preguntó por el número mínimo de personajes en un cuento y los estudiantes tuvieron un $78,8 \%$ de aciertos en la encuesta inicial contra un $81,8 \%$ en la final. 
Para terminar, con la pregunta 11 se quiso averiguar por el destino del héroe en un cuento, $y$ se encontró un $27,3 \%$ de respuestas correctas en un comienzo, el cual descendió a $24,2 \%$ en la encuesta de salida.

En términos numéricos, solo el $9,1 \%$ registró una calificación de 2,7 sobre 5 ; el $42,4 \%$ del grupo obtuvo notas de 3,2 y 3,6, mientras que el 48,5\% restante se ubicó entre 4,1 y 5,0. La nota promedio pasó, entonces, de 3,2, en la encuesta inicial, a 3,9, en la final.

En resumen, el contraste de las encuestas de entrada y salida permitió apreciar que el AT fue útil, ya que los jóvenes lograron reconocer aspectos esenciales de algunas de las etapas del esquema narrativo, como la situación inicial, el desenlace y la situación final, al igual que aprendieron a identificar y emplear los tiempos característicos de los cuentos en francés.

\section{Conclusiones}

Este estudio, como una propuesta de intervención educativa, permitió implementar y evaluar la estrategia didáctica de agrupamiento de textos para la lectura y la escritura de cuentos en el componente de literatura francesa del curso de Habilidades Integradas IV de la Licenciatura en Lenguas Extranjeras de la Escuela de Ciencias del Lenguaje de la Universidad del Valle, cumpliendo así con el objetivo general. Asimismo, los objetivos específicos se cumplieron, gracias a la aplicación de los diferentes instrumentos previstos.

El análisis documental evidenció la falta de objetivos y contenidos explícitos para abordar el componente literario en los programas de los cuatro primeros semestres de formación en FLE. Ante tal evidencia, se propusieron cuatro corpus para integrar la competencia literaria al aprendizaje del francés; estos se presentaron en la tabla 1 .

El AT fue efectivo para que los estudiantes identificaran las etapas y particularidades del cuento en la fase inicial de lectura. Luego, en la fase final, ellos transpusieron tales elementos y lograron redactar sus propios cuentos; el 95,6\% cumplió con la tarea.

Asimismo, los cuentos, producto del proceso de escritura de invención por parte de los aprendientes, fueron insumo suficiente para determinar los avances en la producción escrita y fueron sometidos, además, a procesos de coevaluación y evaluación. Gran parte de los relatos reflejaron originalidad y un manejo lingüístico acorde con las exigencias de este género literario.

Finalmente, la encuesta permitió ver que los estudiantes mejoraron en: la identificación del número de etapas de un cuento corto; la caracterización de la situación inicial, del desenlace y de la situación final de esta tipología, y el reconocimiento de los tiempos propios del relato. Por el contrario, la encuesta no fue efectiva para descubrir el elemento perturbador del inicio de la historia ni para reconocer que el héroe puede terminar bien o mal. Futuros estudios deberían abordar otras secuencias didácticas para que el AT permita mejorar estas falencias.

\section{Referencias}

Adam, J.-M., y Lorda, C.-U. (1999). Lingüistica de los textos narrativos. Barcelona: Ariel.

Alloprof (1996). Français. Le schéma narratiff. Recuperado de http://www.alloprof.qc.ca/BV/pages/f1050.aspx.

Boissinot, A. y Mougenot, M. (1992). Le groupement de textes. Présentation. Le français aujourd'bui, (97), 3-9.

Brunner, D., Vercruyssen, J., Thiltges, P. (Productores), y Ocelot, M. (Dirección). (1998). Kirikou et la sorcière [Película]. Francia: Gébéka Films.

Colombia, Ministerio de Educación Nacional (1998). Lineamientos curriculares lengua castellana. Recuperado de http://www.mineducacion.gov.co/1621/ articles-89869_archivo_pdf8.pdf.

Elliott, J. (1993). El cambio educativo desde la investigaciónacción. Madrid: Ediciones Morata.

Genette, G. (1982). Palimpsestes. La littérature au second degré. Paris : Éditions du Seuil.

Grimm, J., y Grimm, W. (1986). Hansel et Gretel. En G. Padoan, 366 contes merveilleux (pp. 161-163). París: Deux coqs d'or. 
Hamon, P. (1991). La description littéraire. De l'Antiquité à Roland Barthès : une anthologie. Paris : Macula.

Houdart-Merot, V. (2004). Réécriture \& écriture d'invention au lycée. Paris : Hachette Livre.

Jordy, J. (1992). Pour une typologie du groupement de textes. Le français aujourd'hui, (97), 10-16.

Jouve, V. (2006). La poétique du roman. Paris : Armand Collin.

La France, Ministère de l'éducation nationale (2010, 30 septiembre). Programme de l'enseignement commun de français en classe de seconde générale et technologique et en classe de première des séries générales et programme de l'enseignement de littérature en classe de première littéraire. Bulletin officiel spécial $n^{\circ} 9$ du 30 septembre 2010. Recuperado de http://www.education.gouv.fr/cid53318/mene1019760a.html

Lager, E. (2003). Análisis de las nuevas orientaciones de la enseñanza de la literatura en el bachillerato francés. Lenguaje, (31), 186-205.

Larivaille, P. (1974). L'analyse (morpho)logique du récit. Poétique (19), 368-388.

Le Conte Merveilleux (s. a.). Didacticiel. Recuperado de http://alexwohl.chez-alice.fr/jeudecadre.html
Leclair, D. (1992). Autour du poeme: Former de vrais lecteurs (About the Poem: Creating True Readers). Le Français dans le Monde (251), 62-67.

Mekhnache, M. (2010). Le texte littéraire dans le projet didactique : Lire pour mieux écrire. Synergies, (9), 121-132.

Moreno, J. (2001). El agrupamiento de textos. Revista Universidad de Medellin, (73), 111-119.

Ouellet, S., y Boutin, J.-F. (2006). La compétence littéraire pour renouveler l'enseignement de la littérature. Enseigner au collégial, une profession à partager. En $26^{i}$ colloque AQPC (pp. 153-162). Québec : Association québécoise de pédagogie collégiale.

Pineau, M. (2012, 16 de diciembre). Le schéma narratif. Recuperado de http://mpineaupropose.over-blog. com/article-le-schema-narratif-113516754.html

Sandín, M. P. (2003). Investigación cualitativa en educación. Fundamentos y tradiciones. Madrid: McGraw-Hill/ Interamericana de España.

Séoud, A. (1997). Pour une didactique de la littérature. París: Les éditions Didier.

Tournier, M. (2008). La mère Noël. En Sept contes (pp. 92-95). Paris : Gallimard Jeunesse. 


\title{
Anexos
}

\section{Anexo 1}

\author{
Universidad del Valle \\ Escuela de Ciencias del Lenguaje \\ Programa de Lenguas Extranjeras \\ Curso Habilidades Integradas en Francés IV \\ Andrés I. Bernal
}

\section{Hansel et Gretel - Frères Grimm}

Hansel et Gretel est un conte merveilleux des frères Jacob et Wilheim Grimm. Il y a de différentes versions un peu partout dans le monde et par conséquent des publications en différentes langues, des vidéos, du théâtre et des films y compris le récemment produit en 2012 du réalisateur Tommy Wirkola. Les frères Grimm sont philologues et linguistes allemands. Ils sont auteurs de légendes et de contes populaires pour enfants. Parmi leurs contes les plus connus on a : Cendrillon, Le petit Poucet, Blanche neige, La belle au bois dormant.

\section{Atelier}

1. Travaillez en couple et reconstituez l'ordre du récit à l'aide des articulateurs et lisez le conte Hansel et Gretel.

2. Discutez avec votre copain / copine et identifiez le(s) sujet(s) de l'histoire, servez-vous des champs lexicaux.
3. Combien de parties répérez-vous dans le conte ? Numérotez-les sur la copie et résumez chacune des étapes.

4. Répérez les personnages et leur rôle.

5. Soulignez le(s) nouveau(x) temps et comparez-le(s) avec celui ou ceux que vous connaissez déjà surtout au niveau de leur emploi.

\section{Anexo 2}

\section{Universidad del Valle - Escuela de Ciencias del Lenguaje Programa de Lenguas Extranjeras Curso de Habilidades Integradas en Francés IV}

Proyecto: "El agrupamiento de textos, una estrategia transversal para la formación literaria en FLE".

\section{Sondage}

Avec ce sondage on veut explorer vos connaissances préalables à propos de certaines caractéristiques du conte et son schéma narratif.

1. D’après vous, combien d'étapes essentielles composent un conte ?

Lesquelles?

Cochez la lettre que vous croyez répond mieux à la question :

2. Qu'est-ce que le schéma narratif?
a. le destin du héros.
b. les différentes fonctions des personnages.
c. la succession des étapes qui construisent le conte.
d. la façon de raconter un conte.

3. La situation initiale...

a. présente le cadre du conte, le rôle que vont jouer les personnages dans l'histoire.

b. présente le cadre du conte, les personnages, les principales actions qui vont suivre.

c. présente le cadre du conte, les personnages, leurs caractéristiques essentielles.

d. est la partie la plus longue du récit.

4. Quest-ce qui vient perturber la situation initiale?

a. un événement ou un choix du héros.

b. l'action de l'opposant. 
c. l'action du héros.

d. les actions et les événements qui mettent fin aux aventures du personnage principal.

5. Qu'est-ce que l'action, le nœud ou le déroulement ?
a. l'ensemble de personnages.
b. les aides du héros.
c. Les péripéties que le personnage principal doit surmonter.
d. les épreuves vécues par l'opposant.

6. Qu'est-ce qu'introduit la situation finale ?
a. Le retour au point de départ.
b. Un rebondissement de l'action dans le conte
c. Le retour à la maison.
d. Le retour de la stabilité.

7. Les temps les plus employés dans les contes sont ...
a. le présent et l'imparfait.
b. l'imparfait et le passé simple.
c. le présent et le passé simple.
d. le passé simple et le passé composé.

504
a. deux personnages.
b. deux fins possibles.
c. deux opposants.
d. deux héros.

9. Ce que recherche le héros...

a. c'est toujours la même chose.

b. c'est une personne, une chose, une qualité ...

c. c'est toujours un objet.

d. c'est ce qu'on lui a volé.

10. L'aide du héros est...
a. ce qui vient affronter l'opposant et trouver ce qu'il cherche à sa place.
b. ce qu'il recherche.
c. ce qui vient aider le héros à affronter l'opposant, à trouver ce qu'il cherche.
d. une personne qui l'aide.

11. Dans un conte, le destin du héros c'est...
a. de ne jamais échouer.
b. de toujours triompher.
c. d'échouer puis de réussir.
d. de voir sa situation s'améliorer ou bien tourner mal.

12. Un conte montre...
a. comment on peut laisser les choses s'améliorer d'elles même, tant qu'on a sa bonne étoile avec soi.
b. que tout est joué d'avance et qu'on ne peut rien faire pour changer son destin.
c. comment on doit affronter des nombreu- ses épreuves avant de comprendre et de réussir.
d. que la gentillesse et la bonté permettent de surmonter toutes les difficultés.
Merci de votre coopération!

How to reference this article: Bernal Vargas, A. I. (2018). El agrupamiento de textos, una estrategia para la formación literaria en francés como lengua extranjera. Íkala, Revista de Lenguaje y Cultura, 23(3), 487-504. DOI: 10.17533/udea.ikala.v23n03a05 